\title{
THE ALEXANDER POLYNOMIAL AND FINITE TYPE 3-MANIFOLD INVARIANTS
}

\author{
STAVROS GAROUFALIDIS AND NATHAN HABEGGER
}

\begin{abstract}
Using elementary counting methods, we calculate the universal perturbative invariant (also known as the $L M O$ invariant) of a 3-manifold $M$, satisfying $H_{1}(M, \mathbb{Z})=\mathbb{Z}$, in terms of the Alexander polynomial of $M$. We show that +1 surgery on a knot in the 3 -sphere induces an injective map from finite type invariants of integral homology 3 -spheres to finite type invariants of knots. We also show that weight systems of degree $2 m$ on knots, obtained by applying finite type $3 m$ invariants of integral homology 3 -spheres, lie in the algebra of Alexander-Conway weight systems, thus answering the questions raised in $\mathrm{Ga}$.
\end{abstract}

\section{Contents}

1. Introduction 1

\begin{tabular}{ll}
\hline 1.1. & History
\end{tabular}

\begin{tabular}{lll}
\hline 1.2. & Statement of the results & 2
\end{tabular}

\begin{tabular}{lll}
\hline 1.3. & Acknowledgment & 3
\end{tabular}

\begin{tabular}{lll}
\hline 2. & Preliminaries & 4
\end{tabular}

2.1. Preliminaries on Chinese characters 4

2.2. The Alexander-Conway polynomial and its weight system 5

$\begin{array}{lll}2.3 . & \text { Preliminaries on the } L M O \text { invariant } & 7\end{array}$

\begin{tabular}{lcc}
\hline $3 . \quad$ Proofs & 8
\end{tabular}

\begin{tabular}{ll}
\hline References & 10
\end{tabular}

\section{INTRODUCTION}

1.1. History. In their fundamental paper, T.T.Q. Le, J. Murakami and T. Ohtsuki [LMO constructed a map $Z^{L M O}$ which associates to every oriented 3-manifold an element of the graded (completed) Hopf algebra $\mathcal{A}(\emptyset)$ of trivalent graphs.] The restriction of this map to the set of oriented integral homology 3 -spheres was shown in [Le1 to be the universal finite type invariant of integral homology 3-spheres (i.e., it classifies such invariants). Thus $Z^{L M O}$ is a rich (though not fully understood) invariant of integral homology 3 -spheres. However, the invariant $Z^{L M O}$ behaves differently as soon as the first Betti number of the 3-manifold, $b_{1}(M)$, is positive. In [Ha2], the second author used an elementary counting argument to deduce that $Z^{L M O}(M)=1$, if $b_{1}(M)>3$, and to compute $Z^{L M O}(M)$, if $b_{1}(M)=3$ (and

Date: Prliminary version, July 22,1997. This version October 29, 2018.

The authors were partially supported by NSF grant DMS-95-05105 and by the CNRS respectively.

This and related preprints can also be obtained at http://www.math.brown.edu/ stavrosg and at http://www.math.sciences.univ-nantes.fr/preprints/.

${ }^{1}$ For a different construction of $Z^{L M O}(M)$ for a rational homology 3 -sphere $M$, see BGRT2]. 
also for $b_{1}(M)=2$, see [BH]), in terms of the Lescop invariant [Les] of $M$. It is an open problem to compute $Z^{L M O}(M)$, for $b_{1}(M)=0,1$.

It is the purpose of the present paper to exploit elementary counting methods in order to calculate $Z^{L M O}(M)$, for 3-manifolds $M$ which satisfy $H_{1}(M, \mathbb{Z})=\mathbb{Z}$, in terms of a "classical invariant" of $M$, namely its Alexander polynomial. This includes the special case of 0surgery? of a knot $K$ in $S^{3}, S_{K, 0}^{3}$, in which case the Alexander polynomial of $S_{K, 0}^{3}$ is the Alexander-Conway polynomial of $K$. An important ingredient of our computation is the recent result of A. Kricker, B. Spence, and I. Aitchinson, [Kr, KSA], calculating the Conway weight system on Chinese characters.

Although the invariant $Z^{L M O}\left(S_{K,+1}^{3}\right)$, of +1 -surgery on a knot $K$ (in contrast to 0-surgery), is not determined by the Alexander-Conway polynomial of $K$ (there are examples with nontrivial invariant, and trivial Alexander polynomial), we show that after truncating $Z^{L M O}$ at degree $m$, the associated degree $2 m$ knot weight system lies in the algebra of the AlexanderConway weight systems. Similar methods allow us to show that finite type invariants of integral homology 3-spheres are determined by their associated knot invariants, thus answering positively the questions (see below) that were posed in [Ga] prior to the construction of the LMO invariant. (At that time, the only known finite type invariant of 3-manifolds was the Casson invariant.)

1.2. Statement of the results. All 3-manifolds and links considered in the present paper will be oriented.

Theorem 1. Let $M$ be an oriented, closed, connected 3-manifold satisfying $H_{1}(M, \mathbb{Z})=\mathbb{Z}$. The universal invariant $Z^{L M O}(M) \in \mathcal{A}(\emptyset)$ can be calculated in terms of the AlexanderConway polynomial $A(M)$ of the 3-manifold. Conversely, the Alexander polynomial of $M$ can be calculated in terms of the universal invariant $Z^{L M O}(M) \in \mathcal{A}(\emptyset)$.

A precise formula relating the two invariants will be given in section 2 .

We outline here the basic idea of the proof, which though somewhat technical, really is quite elementary: If a manifold $M$ is obtained by 0 -surgery on a knot $K$ in $S^{3}$ (the general case of a manifold satisfying $H_{1}(M, \mathbb{Z})=\mathbb{Z}$ is not much harder), then quite immediately from the definitions, the degree $m$ part of $Z^{L M O}(M)$ can be computed from the part of the Kontsevich integral of $K$ (written in Chinese characters) which has $2 m$ legs and $2 m$ internal vertices. Since there are no components which are intervals (because of the 0-framing), and since by the anti-symmetry relation, all trees vanish, the only contributing part consists of wheels. But this part is known to determine the Alexander polynomial of $K$, and thus that of $M$.

Before we state the next result, we need to recall some standard definitions and notation from the theory of finite type invariants of knots and integral homology 3 -spheres, see [B1, Oh, Ga, LMO].

Let $\mathcal{K}$ denote the vector space over $\mathbb{Q}$ on the set of isotopy classes of oriented knots in $S^{3}$ and let $\mathcal{F}_{m} \mathcal{V}$ (for a nonnegative integer $m$ ) denote the vector space of finite type (i.e.,

\footnotetext{
${ }^{2}$ given a framed link $L$ in a 3-manifold $M$, we denote by $M_{L}$ the result of Dehn surgery on $L$.

${ }^{3}$ An earlier version of this paper contained only this special case. We extend special thanks to C. Lescop, for help in extending to the general case and to D. Thurston, for pointing out that the result should hold in this generality.
} 
Vassiliev) invariants of knots of type $m$, [B1]. Similarly, let $\mathcal{M}$ denote the vector space over $\mathbb{Q}$ on the set of orientation preserving diffeomorphism classes of oriented integral homology 3 -spheres, and let $\mathcal{F}_{m} \mathcal{O}$ denote the vector space of finite type (i.e., Ohtsuki) invariants of integral homology 3-spheres of type $m$, Oh]. In [Ga] we considered the map $K \mapsto S_{K,+1}^{3}$. This is a classical map, often used in the study of knots (or 3-manifolds). This yields a map $\mathcal{K} \rightarrow \mathcal{M}$ and a dual map $\Phi: \mathcal{M}^{*} \rightarrow \mathcal{K}^{*}$ (where $V^{*}$ denotes the dual of a vector space $V$ ). In $\mathrm{Ga}$ the following questions were posed:

Q1: Does the above map send $\mathcal{F}_{3 m} \mathcal{O}$ to $\mathcal{F}_{2 m} \mathcal{V}$ ?

Q2: Is the restriction of the map $\Phi$ to $\mathcal{F}_{3 m} \mathcal{O}$ one-to-one, for all $m$ ?

Q3: Assuming the answer to Question 1 is affirmative, and given $v \in \mathcal{F}_{3 m} \mathcal{O}$, is it true that the associated degree $2 m$ knot weight system lies in the algebra of the AlexanderConway weight systems?

Let $v$ be a $\mathbb{Q}$-valued invariant of integral homology 3-spheres and let $\Phi(v)$ be the associated invariant of knots in $S^{3}$. Question 1 asks whether $\Phi(v)$ is a finite type invariant of knots in $S^{3}$ (together with an estimate of the type of the invariant), if $v$ is a finite type invariant of integral homology 3-spheres. Question 2 asks whether $\Phi(v)$ determines $v$. (It should be noted, however, that there are integral homology 3-spheres that cannot be obtained by \pm 1 surgery on a knot in $S^{3}$, see [A].) Question 3 is concerned with the finite type knot invariant $\Phi(v)$ and asks whether in degree $2 m$ (the maximum possible degree by question 1$), \Phi(v)$ is a classical knot invariant (on elements in the $2 m$-th term of the Vassiliev filtration), given by a polynomial in the Alexander-Conway coefficients.

Building on work of the first author and J. Levine (a preliminary version of [GL2]), Question 1 was answered affirmatively by the second author, [Ha1]. Alternative proofs were later given in GL2, Le1. The methods used in Ha1 and GL2 were a mixture of geometric topology together with a counting argument. On the other hand, [Le1 used the $Z^{L M O}$ invariant and an elementary counting argument.

Using elementary counting arguments similar to those in [Le1], together with properties of the $Z^{L M O}$ invariant, enables us to show that Questions 2 and 3 above are true.

Theorem 2. The association, which takes a knot in $S^{3}$ to the integral homology sphere obtained by +1-framed surgery on the knot, induces an injection from the space of finite type 3-manifold invariants (in the sense of Ohtsuki), to the space of finite type (Vassiliev) knot invariants.

Theorem 3. Let $v$ be a finite type $3 m$ invariant (in the sense of Ohtsuki) of homology 3-spheres. Then the associated degree $2 m$ knot weight system lies in the algebra of the Alexander-Conway weight systems.

Remark 1.1. Theorem 2 does not hold at the graded level, i.e., the associated graded map $\mathcal{G}_{m} \Phi: \mathcal{G}_{3 m} \mathcal{O} \rightarrow \mathcal{G}_{2 m} \mathcal{V}$ is not one-to-one for $m \geq 4$ (see remark 3.2).

1.3. Acknowledgment. We would like to thank Dror Bar-Natan, Vincent Franjou, Jerry Levine, Thang T.Q. Le, Christine Lescop, Gregor Masbaum, Paul Melvin, Xiao-Song Lin, Dylan Thurston and Pierre Vogel for useful conversations. We also wish to thank the referee for numerous suggestions and comments. 


\section{Preliminaries}

2.1. Preliminaries on Chinese characters. Recall that a Chinese character is a graph such that every vertex has valency 1 or 3 (often called a uni-trivalent graph), together with a cyclic order of the edges at each of its trivalent vertices. There is a degree-preserving linear isomorphism $\chi: \mathcal{B} \rightarrow \mathcal{A}\left(S^{1}\right)$ between the graded coalgebra $\mathcal{B}$ of Chinese characters (modulo the antisymmetry and IHX relations) and the graded coalgebra of chord diagrams $\mathcal{A}\left(S^{1}\right)$ on a circle, see [B1, theorem 8], given by mapping a Chinese character $C$ with $n$ legs to $1 / n$ ! times the sum of the $n$ ! ways of joining all of its legs to $n$ chosen ordered points on a fixed circle. The degree of a Chinese character or chord diagram is half the number of vertices, and the primitive diagrams are the connected ones.

Since the map $\chi$ is a vector space isomorphism, we will identify $\mathcal{B}$ and $\mathcal{A}\left(S^{1}\right)$ via $\chi$. Note that $\mathcal{B}$ has two commutative multiplications; 用 one is induced by the multiplication on $\mathcal{A}\left(S^{1}\right)$ via $\chi$, denoted by $\cdot_{\times}$, and the other is the disjoint union of Chinese characters, denoted by $\cdot \sqcup$. In what follows, we will suppress $\cdot \times$ from the notation, but will explicitly use $\sqcup$. Thus, $\exp _{\sqcup}$ will be used to designate the exponential with respect to the $\sqcup$ multiplication, and $\exp$ will be used to designate the exponential with respect to the $\cdot \times$ multiplication.

We will be interested in several important subspaces of $\mathcal{B}$. Let $\mathcal{B}^{\prime}$ denote the subspace of $\mathcal{B}$ which is spanned by Chinese characters, no component of which is (homeomorphic to) an interval. $\mathcal{B}^{\prime}$ is a subalgebra of $\mathcal{B}$ with respect to either multiplication. Note that $\mathcal{B}^{\prime}$ is a direct summand of $\mathcal{B}$ with complementary factor the span of Chinese characters which contain an interval component. $\mathcal{B}^{\prime}$ is related to a deframing projection map $F: \mathcal{A}\left(S^{1}\right) \rightarrow \mathcal{A}\left(S^{1}\right)$ (whose image will be denoted by $\left.\mathcal{A}^{\prime}\left(S^{1}\right)\right)$ defined in [B1, part 2 of Theorem 4 and exercise 3.16]. Using the isomorphism $\chi$, the image of the induced deframing map (also denoted by $F$ ) $F: \mathcal{B} \rightarrow \mathcal{B}$ [ was shown in [KSA, Corollary 4.4] to coincide with $\mathcal{B}^{\prime} \cdot[$

Let $\mathcal{B}^{\prime \prime}$ denote the subspace of $\mathcal{B}$ which is spanned by Chinese characters some component of which has more trivalent vertices than univalent ones. $\mathcal{B}^{\prime \prime}$ is a direct summand of $\mathcal{B}^{\prime}$. In fact, one has the direct sum decomposition $\mathcal{B}^{\prime}=\mathcal{B}^{\prime \prime} \oplus \mathcal{B}_{w h}$, where $\mathcal{B}_{w h}{ }^{\text {[ }}$ denotes the subspace of $\mathcal{B}$ spanned by all Chinese characters every component of which is a wheel (see section 2.2 below).

Let $P_{w h}: \mathcal{B} \rightarrow \mathcal{B}$ denote the composition of the deframing map $F$ followed by the projection to the subspace $\mathcal{B}_{w h}$.

We close this section with the following characterization of the algebra of AlexanderConway weight systems, due to [KSA, $\mathrm{Kr}]$. Recall that a weight system $W$ is a linear map $W: \mathcal{A}\left(S^{1}\right) \rightarrow \mathbb{Q}$. Weight systems can be multiplied and thus they form an algebra. Given a finite type invariant of knots (or a power series of such invariants, such as the AlexanderConway polynomial, which will be discussed in greater detail in the next section) there is an associated weight system, generating a subalgebra in the algebra of weight systems. We now have the following

\footnotetext{
${ }^{4}$ The two multiplications are different. For a conjectural relation between these two multiplications, see [BGRT1, Conjecture 2].

${ }^{5} \mathrm{An}$ interval is a Chinese character of degree 1 with 2 univalent vertices and no trivalent ones.

${ }^{6}$ Note that $F$ is not the projection in the above direct-sum decomposition of $\mathcal{B}$.

${ }^{7}$ As an exercise, the reader may try to find a conjectural formula for $F$ in terms of Chinese characters using [BGRT1, Conjecture 2].

${ }^{8}$ With respect to the $\cdot \sqcup$ multiplication, $\mathcal{B}_{w h}$ is a polynomial algebra on the set of wheels with an even number of legs (the odd-legged wheels vanish by antisymmetry).
} 
Theorem 4. [K], [KSA] A weight system $W: \mathcal{A}\left(S^{1}\right) \rightarrow \mathbb{Q}$ lies in the algebra of AlexanderConway weight systems if and only if it factors through $P_{w h}$.

2.2. The Alexander-Conway polynomial and its weight system. In this section we review some well known properties of the Alexander-Conway polynomial and its associated weight system. (For the Alexander-Conway polynomial and further references, see for example the exposition in the appendix of [Les].) The Conway polynomial $C$ [Co, Ka of a knot (considered as a polynomial in $z$ ) is defined by the relations:

$$
\begin{gathered}
C(Y)-C(\lambda)=z C()(), \\
C(c \text {-component unlink })= \begin{cases}1 & \text { if } c=1 \\
0 & \text { otherwise. }\end{cases}
\end{gathered}
$$

With the terminology of [BG, section 3.1], the Conway polynomial itself is not a canonical Vassiliev power series, but its renormalized reparametrized version

$$
\tilde{C}(\hbar)=\frac{\hbar}{e^{\hbar / 2}-e^{-\hbar / 2}} C\left(e^{\hbar / 2}-e^{-\hbar / 2}\right)
$$

is a canonical Vassiliev power series (i.e., it satisfies $\tilde{C}(K)(h)=W_{C, \hbar} \circ Z^{K}(K)$, see below). Similarly, the Alexander polynomial, defined by $A(t)=C\left(t^{1 / 2}-t^{-1 / 2}\right)$, is not a canonical Vassiliev power series, but it becomes canonical when multiplied by $\frac{\hbar}{e^{\hbar / 2}-e^{-\hbar / 2}}$ and evaluated at $t=e^{\hbar}$ (as this product is $\left.\tilde{C}(\hbar)\right)$.

Let $W_{C}: \mathcal{A}\left(S^{1}\right) \rightarrow \mathbb{Q}$ denote the weight system of $\tilde{C}$ (which is equal to the weight system of $C$ ). It has the property that it is a deframed multiplicative weight system. (Recall that a weight system $W: \mathcal{A}\left(S^{1}\right) \rightarrow \mathbb{Q}$ is called deframed if it factors as a composition $\mathcal{A}\left(S^{1}\right) \stackrel{F}{\rightarrow} \mathcal{A}\left(S^{1}\right) \rightarrow \mathbb{Q}$, where $F$ is the deframing map. Furthermore, a weight system $W: \mathcal{A}\left(S^{1}\right) \rightarrow \mathbb{Q}$ is called multiplicative if for all chord diagrams $C D_{1}, C D_{2}$ of degrees $m_{1}, m_{2}$ respectively, we have: $W_{m_{1}+m_{2}}\left(C D_{1} \cdot C D_{2}\right)=W_{m_{1}}\left(C D_{1}\right) W_{m_{2}}\left(C D_{2}\right)$.)

The weight system $W_{C}$ was calculated on linear chord diagrams in [BG, Theorem 3]. Its expression in terms of Chinese characters in $\mathcal{B}^{\prime}$ was given by Kricker [Kr, Theorem 2.10] as follows:

$$
W_{C}(\xi)= \begin{cases}(-2)^{p} & \text { if } m=2 n \text { and } \xi \text { is a disjoint union of } \\ 0 & p \text { even-legged wheels } \\ 0 & \text { otherwise }\end{cases}
$$

where $\omega_{2 n}$ is a wheel with $2 n$ legs, see Figure 1.

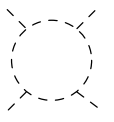

Figure 1. The wheel $\omega_{4}$ with 4 legs. Its trivalent vertices are oriented clockwise.

Let $Z^{K}: \mathcal{K} \rightarrow \mathcal{A}\left(S^{1}\right)$ denote the universal finite type invariant of knots, constructed by Kontsevich [Ko (see B1]), and let $\xi(K) \stackrel{\text { def }}{=} \log Z^{L M O}(K)$ denote its logarithm. (N.b., since 
knots are considered as unframed, their image under the map $Z^{K}$ lies in the image of the deframing map.] For an extension to a functor $Z$ on the category of framed $q$-tangles, see e.g., B2, Ca, [KT, LM1]. Then for a 0 -framed knot $K$ in $S^{3}, Z^{K}(K)$ coincides with the value of $Z$ on $K$. In particular, $\xi(K)$ lies in (the primitive part of, see for example [LM2]) $\mathcal{A}^{\prime}\left(S^{1}\right) \simeq \mathcal{B}^{\prime}$. So $P_{w h} \xi(K)$ consists of a sum of (even-legged) wheels.

Define $a_{2 m}(K)$ by

$$
\sum_{m=1}^{\infty} a_{2 m}(K) \omega_{2 m} \stackrel{\text { def }}{=} P_{w h} \xi(K) .
$$

Let $W_{C, \hbar}$ denote the product of $W_{C}$ and $\hbar^{\text {deg }}$, where $\hbar^{\text {deg }}$ is the operator that multiplies every degree $m$ diagram by $\hbar^{m}$. Let $\mathcal{U}$ denote the (zero-framed) unknot四 and set $b_{2 m}=$ $a_{2 m}(\mathcal{U})$. We now claim that

- Fact 1. For a zero framed knot in $S^{3}$ we have:

$$
-1 / 2 \log \left(A(K)\left(e^{\hbar}\right)\right)=\sum_{m=1}^{\infty} a_{2 m}^{\prime}(K) \hbar^{2 m},
$$

where $a_{2 m}^{\prime}(K)=a_{2 m}(K)-b_{2 m}$. Indeed, one has that

$$
\begin{aligned}
\tilde{C}(K)(h) & =W_{C, \hbar} \circ Z^{K}(K) & & \text { since } \tilde{C} \text { is canonical } \\
& =\exp \left(W_{C, \hbar} \xi(K)\right) & & \text { since } W_{C, \hbar} \text { is multiplicative } \\
& =\exp \left(-2 \sum_{m=1}^{\infty} a_{2 m}(K) \hbar^{2 m}\right) & & \text { by equation (2). }
\end{aligned}
$$

Thus we have, $-1 / 2 \log (\tilde{C}(K)(\hbar))=\sum_{m=1}^{\infty} a_{2 m}(K) \hbar^{2 m}$. In particular, $-1 / 2 \log \left(\frac{\hbar}{e^{\hbar / 2}-e^{-\hbar / 2}}\right)=$ $\sum_{m=1}^{\infty} b_{2 m} \hbar^{2 m}$, and the result follows since $\tilde{C}(K)(\hbar)=\tilde{C}(\mathcal{U})(\hbar) A(K)\left(e^{\hbar}\right)$.

Define $\alpha(K)$ in (the completion of) $\mathcal{B}_{w h}$ by:

$$
\alpha(K)=\sum_{m=1}^{\infty}\left(b_{2 m}+a_{2 m}(K)\right) \omega_{2 m}
$$

Note that $\alpha(K)=\sum_{m=1}^{\infty}\left(2 b_{2 m}+a_{2 m}^{\prime}(K)\right) \omega_{2 m}$.

Similarly, let $M$ denote a 3-manifold which satisfies $H_{1}(M, \mathbb{Z})=\mathbb{Z}$, and let $A(M)(t)$ denote its Alexander polynomial, normalized so that it is symmetric (in $t$ and $t^{-1}$ ) and evaluates to 1 at $t=1$. We define $a_{2 m}^{\prime}(M)$ by $-1 / 2 \log \left(A(M)\left(e^{\hbar}\right)\right)=\sum_{m=1}^{\infty} a_{2 m}^{\prime}(M) \hbar^{2 m}$.

Define $\alpha(M)$ by:

$$
\alpha(M) \stackrel{\text { def }}{=} \sum_{m=1}^{\infty}\left(2 b_{2 m}+a_{2 m}^{\prime}(M)\right) \omega_{2 m} .
$$

Obviously, $\alpha(M)$ and $A(M)$ can be computed from each other.

\footnotetext{
${ }^{9}$ Actually, $Z^{K}(K)$ lies in a quotient of $\mathcal{A}\left(S^{1}\right)$ isomorphic to $\mathcal{A}^{\prime}\left(S^{1}\right)$.

${ }^{10}$ It may be of interest to note that the value of $Z^{K}(\mathcal{U})$ is conjecturally given by the equation $P_{w h}(\xi(\mathcal{U}))=$ $\xi(\mathcal{U})$. (See BGRT1, Conjecture 1], where it is shown that the conjecture holds on the level of semisimple Lie algebras.) Note also that the Alexander-Conway polynomial is determined by the $\mathfrak{s l}_{N}$ colored Jones polynomial (see $[B G]$ ).
} 
2.3. Preliminaries on the $L M O$ invariant. In this section we review some well known properties of the invariant $Z^{L M O}$. We denote by $\{x\}_{m}$ the degree $m$ part of $x$. Recall from [LMO that for every integer $f$, and every knot $K$ in $S^{3}$, the value of the universal invariant on $S_{K, f}^{3}$ is given by:

$$
Z^{L M O}\left(S_{K, f}^{3}\right)=\sum_{m=0}^{\infty}\left\{\frac{\iota_{m}\left(c(f) Z^{K}(K)\right)}{c_{m}(f)}\right\}_{m} \in \mathcal{A}(\emptyset)
$$

(the product in $\mathcal{B}$ is taken with respect to the $\cdot_{\times}$multiplication), where $c(f)=\exp \left(\frac{f}{2} \Theta\right) Z^{K}(\mathcal{U})$ and $c_{m}(f)=\iota_{m}\left(\exp (\operatorname{sgn}(f) \Theta / 2) Z^{K}(\mathcal{U})^{2}\right)($ resp. 1) if $f \neq 0$ (resp. $f=0$ ). Here $\Theta$ denotes the unique chord diagram of degree 1 on a circle, $\iota_{m}: \mathcal{A}\left(S^{1}\right) \rightarrow \mathcal{A}(\emptyset)$ is a map defined in [LMO, section 2], $\mathcal{U}$ is the zero framed unknot, and $Z^{K}(K)$ is the value of the universal knot invariant with the zero framing. Note that $Z^{K}(\mathcal{U})$ is denoted by $\nu$ in [LMO, Le1].

Let $\pi_{m}$ denote the projection $\mathcal{A}(\emptyset) \rightarrow \mathcal{A}_{\leq m}(\emptyset)$. In the special case when $f=+1$ (n.b., the formula below holds since we are in the case of an integral homology sphere, see [LMO]), one has the formula

$$
\pi_{m}\left(Z^{L M O}\left(S_{K,+1}^{3}\right)\right)=\pi_{m}\left(\frac{\iota_{m}\left(c Z^{K}(K)\right)}{c_{m}}\right) \in \mathcal{A}_{\leq m}(\emptyset)
$$

where $c=\exp \left(\frac{1}{2} \Theta\right) Z^{K}(\mathcal{U})$ and where $c_{m}=\iota_{m}\left(\exp \left(\frac{1}{2} \Theta\right) Z^{K}(\mathcal{U})^{2}\right)$.

The map $\iota_{m}$, though rather complicated when evaluated on chord diagrams on a circle, becomes more transparent when evaluated on Chinese characters. In particular, it follows from its definition that for a Chinese character $C$ with $l$ legs, we have:

$$
\iota_{m}(C)= \begin{cases}O_{-2 m}(\langle C\rangle) & \text { if } l=2 m \\ 0 & \text { otherwise }\end{cases}
$$

where $\langle C\rangle$ denotes the closure of $C$, i.e., the sum of all $(2 m-1)$ !! ways of closing its legs by joining the univalent vertices in pairs, and $O_{-2 m}$ is the map which sets circle components equal to $-2 m$. Note that in the special case that no connected component of $C$ is an interval $I$, then no connected component of $\langle C\rangle$ is a circle, and so $O_{-2 m}(\langle C\rangle)=\langle C\rangle$. Note also that if $C$ has $2 k$ legs, then $O_{-2 m}(\langle C \cdot \sqcup I\rangle)=(-2)(m-k) O_{-2 m}(\langle C\rangle)$.

We have the following:

- Fact 2. Fix a nonnegative integer $m$. Given $a \in 1+\mathcal{B}_{\geq 1}, b \in \mathcal{B}_{\geq 2 m}^{\prime}, c \in 1+\mathcal{A}_{\geq 1}(\emptyset)$, then:

$$
\left\{\frac{\left.\iota_{m}(a b)\right)}{c}\right\}_{m}=\left\langle P_{w h}\left(\{b\}_{2 m}\right)\right\rangle \in \mathcal{A}_{m}(\emptyset) .
$$

Note that this identity holds with respect to either multiplication in $\mathcal{B}$.

For the proof, note that $\iota_{m}$ reduces degree by $m$. In particular, the only part of $b$ which contributes to both sides is $\{b\}_{2 m}$, the degree $2 m$ part of $b$ lying in $\mathcal{B}_{2 m}^{\prime}$. Note also that an element of $\mathcal{B}_{2 m}^{\prime}$ has at most $2 m$ legs; moreover, it has exactly $2 m$ legs if and only if it lies in $\left(\mathcal{B}_{w h}\right)_{2 m}$, i.e., it is a linear combination of Chinese characters, all of whose components are even-legged wheels. This shows the above claim.

\footnotetext{
11 To see this, see Le2, note that the total symmetrization of the element $T_{l}^{m}$, described in LMO], vanishes, if $l$ is different from $2 m$. The formula follows, since the total symmetrization of $T_{2 m}^{m}$, applied to a Chinese character, corresponds to the sum of all ways of closing up the character.
} 


\section{Proofs}

In this section we give the proofs of theorems 1, 2 1 and 3 .

Lemma 3.1. The map $x \mapsto\langle x\rangle$ from $\mathcal{B}_{w h} \rightarrow \mathcal{A}(\emptyset)$ is injective, but its restriction $\left(\mathcal{B}_{w h}\right)_{2 n} \rightarrow$ $\mathcal{A}_{n}(\emptyset)$ for $n \geq 4$, is not surjective.

Proof. Note that the map \langle\rangle sends connected Chinese characters to connected trivalent graphs. In particular it induces a map of primitives of $\mathcal{B}_{w h}$ which is easily seen to be injective. [2]

Now note further that the map \langle\rangle , although not multiplicative, sends a product of primitives to the product of their closures, plus terms with fewer connected components. This implies that the map \langle\rangle on $\mathcal{B}_{w h}$ is injective, and further that the preimage of the set of primitives is the set of primitives of $\mathcal{B}_{w h}$. Thus, were the map also surjective as well, it would send the primitives onto the primitives. But as the primitive part of $\left(\mathcal{B}_{w h}\right)_{2 n}$ is of dimension 1 , and since the dimension of the primitive part of $\mathcal{A}_{n}(\emptyset)$ is $>1$, for $n \geq 4$ (see e.g. [B1]), it follows that \langle\rangle is not surjective in degree $n \geq 4$.

Proof of theorem [1. We first give the proof in case $M=S_{K, 0}^{3}$ is obtained by surgery on a zero-framed knot $K$ in $S^{3}$. We have

$$
\begin{aligned}
Z^{L M O}(M) & =\sum_{m=0}^{\infty}\left\{\iota_{m}\left(Z^{K}(\mathcal{U}) Z^{K}(K)\right)\right\}_{m} \\
& =\sum_{m=0}^{\infty}\left\{\iota_{m}(\exp (\xi(\mathcal{U})) \exp (\xi(K)))\right\}_{m} \\
& =\sum_{m=0}^{\infty}\left\{\iota_{m}(\exp (\xi(\mathcal{U})+\xi(K)))\right\}_{m} \\
& =\left\langle P_{w h} \exp (\xi(\mathcal{U})+\xi(K))\right\rangle \\
& =\left\langle\exp _{\sqcup}\left(P_{w h}(\xi(\mathcal{U})+\xi(K))\right)\right\rangle \\
& \left.=\left\langle\exp _{\sqcup}\left(\sum_{m=1}^{\infty}\left(a_{2 m}(\mathcal{U})+a_{2 m}(K)\right) w_{2 m}\right)\right)\right\rangle \\
& =\left\langle\exp _{\sqcup} \alpha(K)\right\rangle
\end{aligned}
$$

where the first and second equality is by definition, the third follows since we are in a commutative algebra, the fourth follows from fact 2 (with $a=c=1$ ), the fifth follows since $P_{w h}$ is an algebra homomorphism, and the last two follow from the definitions. This shows that the invariant $Z^{L M O}\left(S_{K, 0}^{3}\right)$ is determined by the Alexander polynomial $A(K)$. Since $A(K)=A(M)$ (and hence $\alpha(K)=\alpha(M)$ ), the result follows.

\footnotetext{
${ }^{12}$ Indeed, consider the multiplicative map $W: \mathcal{A}(\emptyset) \rightarrow \mathbb{Q} \llbracket c \rrbracket$ defined by imposing the relation $W(H)=$ $W(=)-W(X)$ and setting any resulting circle components equal to $c$. Here $H$ denotes a diagram which in a neighborhood of some arc looks like an $H$, and $=$, resp. $X$, is obtained from $H$ by replacing this neighborhood by two arcs joining the 4 points on the boundary of the neighborhood which are on the same side of the arc, resp. diametrically opposed. Then one has that $W\left(\left\langle w_{2}\right\rangle\right)=c^{2}-c$ and that $W\left(\left\langle w_{2 m+2}\right\rangle\right)=(c+2 m) W\left(\left\langle w_{2 m}\right\rangle\right)$, so $\left\langle w_{2 m}\right\rangle \neq 0$.
} 
Conversely, by Lemma 3.1, the map $\sum_{m=1}^{\infty} c_{2 m} w_{2 m} \rightarrow\left\langle\exp _{\sqcup}\left(\sum_{m=1}^{\infty} c_{2 m} w_{2 m}\right)\right\rangle$ is the composite of two injective maps and hence is injective. It follows that $Z^{L M O}\left(S_{K, 0}^{3}\right)$ determines the Alexander polynomial.

To prove the general case, first note that we may obtain $M$ via surgery on a boundary link $K \cup L$ in $S^{3}$, where the framing on $K$ is the zero framing, and the framing of each component of $L$ is \pm 1 . Indeed, one may obtain $M$ by zero-framed surgery on a knot in an integral homology sphere, which in turn may be obtained by surgery on a \pm 1 -framed boundary link $L$. It suffices to isotope, in this homology sphere, the Seifert surface for the knot so as to be disjoint from the Seifert surfaces of the components of $L .^{\text {T3 }}$

In this case, one still has $A(K)=A(M)$ (since the link is a boundary link, the Seifert form on $K$ in $S^{3}$ is the same as the Seifert form of $K$ in the homology sphere obtained by surgery on $L$ ). Moreover, since the link is boundary, its Milnor invariants vanish, and hence, by [HM], $Z^{K}\left(K \cup L_{0}\right)$ consists of diagrams, none of which are trees (where $L_{0}$ denotes the link $L$ with zero framing). Consequently, using a counting argument similar to [Ha2], one can check that: $\iota_{m}\left(c^{\prime}(f) Z^{K}\left(K \cup L_{0}\right)\right)=\iota_{m}\left(c^{\prime}(f) Z^{K}\left(K \sqcup L_{0}\right)\right)$ where $c^{\prime}(f)$ are terms that depend on the framing of $L$, and $K \sqcup L_{0}$ denotes the disjoint union of the links $K$ and $L_{0}$. The definition of the $L M O$ invariant and its multiplicative property under connected sum implies that $Z^{L M O}(M)=Z^{L M O}\left(S_{K, 0}^{3} \sharp S_{L}^{3}\right)=Z^{L M O}\left(S_{K, 0}^{3}\right)$, thus finishing the proof of the theorem.

Proof of theorem [8. Recall, [Le1], that $Z_{\leq m}^{L M O}: \mathcal{M} / \mathcal{M}_{3 m+1} \rightarrow \mathcal{A}_{\leq m}(\emptyset)$ is an isomorphism. We will prove that the map $\mathcal{K} \rightarrow \mathcal{M} / \mathcal{M}_{3 m+1}$ is onto (or equivalently, that the composite map $\mathcal{B}^{\prime} \rightarrow \mathcal{B}_{\leq 2 m}^{\prime}=\mathcal{K} / \mathcal{K}_{2 m+1} \rightarrow \mathcal{M} / \mathcal{M}_{3 m+1}=\mathcal{A}_{\leq m}(\emptyset)$ is onto), which is dual to the statement of theorem 2 .

The map $\mathcal{B}_{\leq 2 m}^{\prime} \rightarrow \mathcal{A}_{\leq m}(\emptyset)$ is given by the formula

$$
x \mapsto \pi_{m}\left(\frac{\iota_{m}(c x)}{c_{m}}\right) \in \mathcal{A}_{\leq m}(\emptyset)
$$

where $c, c_{m}$ are as in secion 2.3 .

Let $x$ be a Chinese character with $2 k$ legs of degree $n+k$, having no interval components, $n \leq m, k \leq m$. Then $\langle x\rangle$ has degree $n$. Under the above mapping, a computation shows that $x \mapsto(-1)^{k}\langle x\rangle+o(n+1)$, where $o(n+1)$ denotes terms of degree $\geq n+1$. Note that any connected graph is the closure of a connected Chinese character with 2 legs. Moreover, the map $x \mapsto\langle x\rangle$ sends a product of connected Chinese characters (without interval components) to the product of their closures plus terms each of which has fewer components. It follows by downward induction on the degree and upward induction on the number of components, that the map $\mathcal{B}^{\prime} \rightarrow \mathcal{A}_{\leq m}(\emptyset)$ is surjective.

Proof of theorem 3. Consider the map $\Psi: \mathcal{K} \rightarrow \mathcal{A}(\emptyset)$ given by:

$$
\Psi(K)=Z^{L M O}\left(S_{K,+1}^{3}\right)
$$

\footnotetext{
13 The same argument can be used to see that every integral homology sphere, $\Sigma$, can be obtained by unit-framed surgery on a boundary link: First note that $\Sigma$ is surgery on some link and after stabilization and handle sliding, the link may be assumed to be \pm 1 -framed with zero linking numbers. In particular, $\Sigma$ can be obtained by a sequence of \pm 1 -framed surgeries on knots in homology spheres. Arguing by induction and applying the Seifert surface argument above, establishes the result.
} 
as well as its truncation, $\Psi_{\leq m}=\pi_{m} \circ \Psi$, where $\pi_{m}$ is the projection $\mathcal{A}(\emptyset) \rightarrow \mathcal{A}_{\leq m}(\emptyset)$. Let $S$ be a $K$-admissible $2 m$-component set. Here $S \subset S^{3}$ denotes the union of $2 m$ disjoint embedded balls that intersect the knot in a \pm crossing, and $[K, S]$ is the signed sum of all knots obtained by changing the crossings. (Recall that such sums generate the $2 m$-th term of the Vassiliev filtration.) One has that:

$$
\begin{aligned}
\Psi_{\leq m}([K, S]) & =\pi_{m}\left(\sum_{S^{\prime} \subseteq S}(-1)^{\left|S^{\prime}\right|} Z^{L M O}\left(S_{K_{S^{\prime}},+1}^{3}\right)\right) \\
& =\pi_{m}\left(\frac{\iota_{m}\left(c Z^{K}([K, S])\right)}{c_{m}}\right) \\
& =\left\{\frac{\iota_{m}\left(c Z^{K}([K, S])\right)}{c_{m}}\right\}_{m} \\
& =(-1)^{m}\left\{\left\langle P_{w h}\left(Z^{K}([K, S])\right)\right\rangle\right\}_{m}
\end{aligned}
$$

(since by [LMO], $\left.\left\{c_{m}\right\}_{0}=(-1)^{m}\right)$.

Similar computations show that $\Psi_{\leq m}([K, S])$ vanishes, if $S$ is a $K$-admissible $n$-component set, with $n>2 m$. It follows that $\Psi_{\leq m}$ is a $\mathcal{A}_{\leq m}(\emptyset)$-valued finite type invariant of knots of order $2 m$. Moreover, the above shows that the weight system $W_{\Psi, m}: \mathcal{A}_{2 m}^{\prime}\left(S^{1}\right) \rightarrow \mathcal{A}_{m}(\emptyset)$ factors through the projection $P_{w h}$ to $\mathcal{B}_{w h}$. It follows from Theorem that $W_{\Psi, m}$ lies in the algebra of Alexander-Conway weight systems with values in $\mathcal{A}(\emptyset)$.

Remark 3.2. The above formula shows that the weight system is explicitly calculated as the composite of the projection to $\left(\mathcal{B}_{w h}\right)_{2 m}$ followed by the closure mapping \langle\rangle$:\left(\mathcal{B}_{w h}\right)_{2 m} \rightarrow$ $\mathcal{A}_{m}(\emptyset)$. This proves the statement dual to that of Remark 1.1, since by Lemma 3.1, the closure mapping on $\mathcal{B}_{w h}$ is injective, but not surjective.

\section{REFERENCES}

[A] D. Auckly, Surgery numbers of 3-manifolds, a hyperbolic example, Geometric topology, Athens GA (1993) 21-32.

[B1] D. Bar-Natan, On the Vassiliev knot invariants, Topology, 34 (1995) 423-472.

[B2] D. Bar-Natan, Non-Associative Tangles, In: Geometric Topology (W. Kazez, Ed.), Proc. Georgia Int. Topology Conf. 1993, AMS/IP Studies in Advanced Mathematics, 1997.

[BG] D. Bar-Natan, S. Garoufalidis, On the Melvin-Morton-Rozansky conjecture, Inventiones, 125 (1996) 103-133.

[BGRT1] D. Bar-Natan, S. Garoufalidis, L. Rozansky, D. Thurston, Wheels, wheeling and the Kontsevich integral of the unknot, preprint, February 1997.

[BGRT2] — The Arhus invariant of rational homology 3-spheres I,II: A highly non trivial flat connection on $S^{3}$, to appear in Selecta Math.

[BH] A. Beliakova, N. Habegger, The Casson-Walker-Lescop Invariant as a Quantum 3-manifold Invariant, preprint July 1997.

[BZ] G. Burde, H. Zieschang, On knots, de Gruyter Studies in Mathematics 5, Berlin, 1985.

[Ca] P. Cartier, Construction combinatoire des invariants de Vassiliev-Kontsevich des nœuds, C. R. Acad. Sci. Paris 316, 1993, 1205-1210.

[CP] V. Chari, A. Pressley, Quantum groups, Cambridge University Press, Cambridge 1994.

[Co] J. H. Conway, An enumeration of knots and links and some of their algebraic properties, in Computational Problems in Abstract Algebra, 329-358, Pergamon, New-York 1970.

[Ga] S. Garoufalidis, On finite type 3-manifold invariants I, J. Knot Theory and its Ramifications 5, no. 4 (1996) 441-462. 
[GL1] S. Garoufalidis, J. Levine, On finite type 3-manifold invariants II, Math. Annalen, 306 (1996) 691-718.

[GL2] - On finite type 3-manifold invariants IV: comparison of definitions, Proc. Camb. Phil. Soc, 122 (1997) 291-300.

[GO] S. Garoufalidis, T. Ohtsuki, On finite type 3-manifold invariants III: manifold weight systems, Topology, 37 (1998) 227-244.

[Ha1] N. Habegger, Finite type 3-manifold invariants: a proof of a conjecture of Garoufalidis, preprint, July 1995.

[Ha2] _ A Computation of the Universal Quantum 3-manifold Invariant for Manifolds of Rank Greater than 2, preprint, December 1996.

[HM] N. Habegger, G. Masbaum, The Kontsevich Integral and Milnor's Invariants, preprint, April 1997.

[Ka] L. H. Kauffman, On knots, Princeton Univ. Press, Princeton, 1987.

[KT] C. Kassel and V. Turaev, Chord diagram invariants of tangles and graphs, University of Strasbourg preprint, January 1995.

[Ko] M. Kontsevich, Vassiliev's knot invariants, Adv. in Sov. Math., 16(2) (1993) 137-150.

[Kr] A. Kricker, Alexander-Conway limits of many Vassiliev invariants, J. Knot Theory and its Ramifications 6 no. 5, (1997) 687-714.

[KSA] A. Kricker, B. Spence, I. Aitchinson, Cabling the Vassiliev invariants, J. Knot Theory and its Ramifications 6 no. 3, (1997) 327-358.

[LM1] T.T.Q. Le, J. Murakami, The universal Vassiliev-Kontsevich invariant for framed oriented links, Compositio Math. 102, 1996, 41-64.

[LM2] - The parallel version of the Kontsevich integral, J. Pure and Applied Algebra, 121 (1997), 271-291.

[LMO] T.T.Q. Le, J. Murakami, T. Ohtsuki, A universal perturbative invariant of 3-manifolds, Topology, 37 (1998) 539-574.

[Le1] T.T.Q. Le, An invariant of integral homology 3-spheres which is universal for all finite type invariants, Soliton Geometry and Topology: On the crossroad, AMS Translations 2 Eds. V. Buchstaber, S. Novikov, $75-100$.

[Le2] - On Denominators of the Kontsevich Integral and the Universal Perturbative Invariant of 3-manifolds, preprint q-alg 9704017.

[Les] C. Lescop Global surgery formula for the Casson-Walker invariant, Annals of Math Studies, 140 Princeton Univ. Press 1996.

[Oh] T. Ohtsuki, Finite type invariants of integral homology 3-spheres, J. Knot Theory and its Rami. 5 (1996) 101-115.

[V] P. Vogel, Algebraic structures on modules of diagrams, preprint August 1995.

Department of Mathematics, Brandeis University, Waltham, MA 02254-9110, U.S.A.

E-mail address: stavros@oscar.math.brandeis.edu

UMR 6629 du CNRS, Université de Nantes, Département de Mathématiques, 2 Rue de la Houssinière, 44072 NANTES Cedex 03, France

E-mail address: habegger@math.univ-nantes.fr 